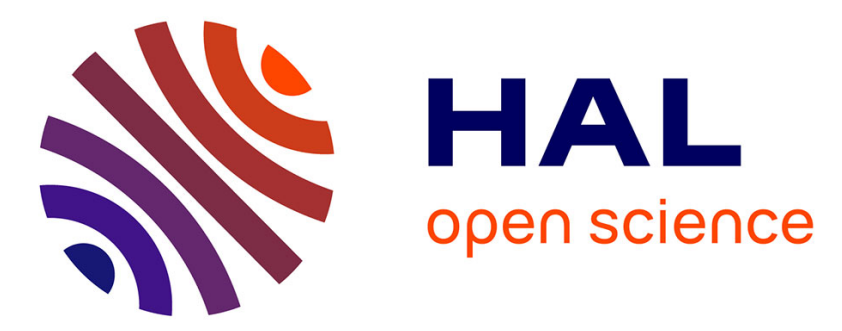

\title{
Controller design for a class of delayed and constrained systems. Application to supply chains
}

\author{
Charifa Moussaoui, Rosa Abbou, Jean-Jacques Loiseau
}

\section{To cite this version:}

Charifa Moussaoui, Rosa Abbou, Jean-Jacques Loiseau. Controller design for a class of delayed and constrained systems. Application to supply chains. A. Seuret; H. Özbay; C. Bonnet; H. Mounier. LowComplexity Controllers for Time-Delay Systems, 2, Springer, 2014, Advances in Delays and Dynamics. hal-01699865

\section{HAL Id: hal-01699865 https://hal.science/hal-01699865}

Submitted on 2 Feb 2018

HAL is a multi-disciplinary open access archive for the deposit and dissemination of scientific research documents, whether they are published or not. The documents may come from teaching and research institutions in France or abroad, or from public or private research centers.
L'archive ouverte pluridisciplinaire HAL, est destinée au dépôt et à la diffusion de documents scientifiques de niveau recherche, publiés ou non, émanant des établissements d'enseignement et de recherche français ou étrangers, des laboratoires publics ou privés. 


\title{
Controller design for a class of delayed and constrained systems. Application to supply chains.
}

Charifa Moussaoui, Rosa Abbou and Jean Jacques Loiseau

\begin{abstract}
This chapter aims to investigate the construction of efficient controllers for some input time delay systems, subjected to strict constraints of positivity and saturating limitations, in presence of some exogenous bounded disturbances. The results are presented through the application of supply chains, for which controller design is a challenging issue trading-off between stabilising properties in presence of time lags, and constraints due to the physical limitations and specificities of the plants of the supply chain. We show that the stabilization of such flow systems can be tackled by the stabilization of input time delay systems, using a predictor based feedback approach. This classical control method, which permits to overcome the delays, is enriched by using saturation terms that allow the consideration of the physical constraints of the system resources composing the serial supply chains.
\end{abstract}

\section{Introduction}

Input time delay systems are common models widely used for dead-time systems representation. Such systems are characterized by the presence of some irreducible time lags, due to, for example, process durations, mass or information transport phenomena and sensor responses. These systems attracted a great deal of attention from both practitioners and researchers, since they are involved in many industrial processes and applications. The control problems related to are challenging issues which fuel constantly the researchers community. Indeed, in addition to the presence of time-lags, these systems are frequently subject to some physical constraints and additional specificities. Furthermore, they are often subject to some exogenous perturbations effects, that makes the controller design task quite more difficult.

Charifa Moussaoui, Rosa Abbou and Jean Jacques Loiseau

LUNAM Université, Institut de Recherche en Communications et Cybernétique de Nantes, UMR 6397. École Centrale de Nantes, 1 rue de la Noë, BP 92101, 44321 Nantes Cedex 3, FRANCE. e-mail: \{Charifa.Moussaoui, Rosa.Abbou, Jean-Jacques.Loiseau\} @irccyn.ec-nantes.fr 
Our concerns focus on the supply lines supply lines which are quite representative of such dead-time systems. They consist of a network of interconnected stages, composed of manufacturers, suppliers parts, exchanging goods, financial and information flows, through transportation, warehousing and retailing operations, all in the sake of fulfilling end-customer requests. These operations are time consuming and request important time-lags that can not be neglected or simply approximated. A central issue is to coordinate them over different stages and locations, while providing a convenient service level to end-customers. This task is quietly enhanced when the market demands are unstable or unknown in advance. In addition, the supply line resources are limited by the storage and the production or supplying capacities, which are current bottlenecks for the system. Indeed, the storage devices, say the inventories, are finite resources that can be subject to congestion problems which lead to important goods losses, while the production units are subject to saturation phenomena due to actuator limitations. These practical problems are commonly performed in other systems, where the dynamics is governed by constrained flow exchanges, such as the communication networks and some others load-balancing systems, where the information flows and the buffers in the network nodes, can be perceived, respectively, as the good flows and the inventories of a supply line.

In this chapter, supply line control issue is formulated as a general constrained control problem, for input time delay systems with positivity constraints and saturated resources, subject to unknown but bounded disturbances. To handle this problem, we make use of predictive-based control techniques, which efficiency in compensating the input time delay is well-established and widely described in the literature. Using pole assignment principle and model reduction [1, 12, 13], we propose a saturated and constrained control law, which allows the controller to handle the system constraints and to meet its specifications. The proposed methodology for designing such controller consists in defining an invariant set for the system trajectories, such that the bounded input bounded output (BIBO) stability property is ensured, and for which the system constraints are meet.

After introducing the problematic, the chapter is organized as follows. In Section 2 , the dynamical model of each stage of the considered supply line is proposed, and a quick review of the literature concerning this topic is presented. Section 3 is devoted to the controller structure, where some backgrounds about saturated commands and the predictive control methods for input time delay systems are presented. In Section 4, the controller design issues are addressed and these results are extended to the case of a multi-level supply line in the Section 5, and illustrated through an numerical example in Section 6. Finally, a discussion about the obtained results and further investigations conclude this chapter.

\section{Problem statement: Inventory and production control}

A supply line is a series of stages or levels, that represents manufacturers, suppliers, transporters and other parts that are involved in supplying process, in which the 
goods flow linearly to reach end-customers, as depicted in Figure 1.

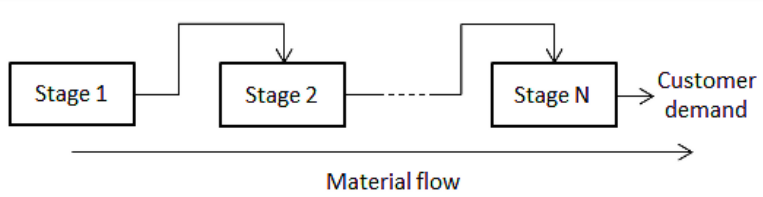

Fig. 1 Multi stages supply chain scheme.

Supply chain control consists of defining appropriate ordering policies that regulate the production and the supply rates in the different stages of the supply line, so that each stage is able to meet the inventories requirements, and to provide a good service level for the incoming demands. In this field, different frameworks where proposed during the past decades, based on optimization procedures using programming techniques, empirical experiences and control theory methods. Our concern focused on the use of the control theory methods, which provide an analytical and formal framework and allow a structural approach to handle the supply chain issues. Indeed, since the pioneering works of Simon [18], who was the first to use Laplace transform to analyze a supply line dynamics, numerous investigations followed, such that $[6,24,5,22,15,26]$, in which the supply chain was modelled using block diagrams and controlled through feedback structures. These investigations lead to the well-known Automatic Pipeline Inventory and Order Based Production Control System (APIOBPCS) models and their variations [10]. They permit to understand the complex interactions that govern supply chains dynamics, identifying the critical agents that impact the inventories stability, such that the delays. The authors highlighted the importance of the Work In Process (WIP), which is the amount of goods ordered in the pipeline but not yet received due to the delay. They also reveal its central role in damping the variance of the demand amplification among the supply chain stages, which is known as the bullwhip or Forrester effect [6].

The advances in the time delay systems control [14, 21], allow further insights into the delayed differential equations describing, in particular, the inventory dynamics $[25,19,4]$, and notable works like [20] permit great extensions considering multiple delays. Nevertheless, the aforementioned works did not take into account the positivity and the capacity constraints of the supply chain resources. Actually, both inventory levels and replenishment orders are constraint free, and are allowed to get some negative values or excessive huge ones, which does not correspond to real plant capacities and thus creates a major gap between theoretical attempts and practical results. For such issues, simulation based analyses are the mostly used, such as in $[3,7,8,29,2,17]$, where the impact of constrained production capacity is studied. In $[27,28]$ an analytical investigation is presented for the forbidden-return case, which corresponds to the constraint of non-negativity on the replenishment orders only. These studies pointed out that considering capacity constrained on the supplying devices, removes the linearity assumption of the model and hence com- 
plex dynamics behaviours are revealed. To the best of our knowledge, no work in this field considers capacity and positivity constraints, on both the supplying devices and the inventories, taking into account the pure delays present in these systems dynamics. This is what this work contributes to. We consider a multi-stage supply line. In a first attempt, our analysis will be held for a single stage, the general case will be presented in Section 5 .

Each stage of the supply line represents an elementary system composed of a supplying unit and a storage one. The term "supplying" is used for the operations of material acquisition, which can be production, transport or retailing process. The supplying units are characterized by a delay $\theta$ which corresponds to the time needed to complete the supplying task, and to a supplying order rate denoted $u(t)$, which is limited by a maximum supplying capacity denoted $U_{\max }$. The storage units are namely the inventories. Each elementary stage of the supply chain has an inventory with a maximum storage capacity denoted $Y_{\max }$. In this work, the customer demands are unknown in advance but assumed to be upper bounded by an amount denoted $d_{\text {max }}$. The generic model for the inventory level dynamics is then described by the following first order delayed equation.

$$
\dot{y}(t)= \begin{cases}u(t-\theta)-d(t) & \text { for } t \geq \theta \\ \phi(t)-d(t) & \text { for } 0 \leq t<\theta,\end{cases}
$$

where, $y(t)$ is the inventory level and $d(t)$ the incoming demand rate of each level. The function $\phi(t)$ describes the initial state of the system such that equation (1) describes the initial dynamics of the inventory for $0 \leq t<\theta$.

As already mentioned, supplying units and inventories as well, are limited resources, which can take non-negative values only. These constraints are formulated as follows. For inventory level

$$
y(t) \in\left[0, Y_{\max }\right], \text { for } t \geq 0,
$$

and for the supplying rate

$$
u(t) \in\left[0, U_{\max }\right], \text { for } t \geq 0
$$

Then, the working assumption on the consumer demand is formulated such as

$$
d(t) \in\left[0, d_{\max }\right], \text { for } t \geq 0 .
$$

The controller design task consists of defining a controller which will stabilize the delayed system (1) while ensuring the fulfilment of the constraints (2) and (3), for every bounded disturbance verifying the assumption (4). 


\section{System control structure}

A local management strategy is used for the elementary level, which aims at meeting its local specifications. In this work, we consider that all stages of the considered supply line are applying the same ordering rules, which consists of fulfilling on line the consumer demands, and replenishing the inventory to a referential level denoted $y_{c}$. The strategy to define on line the control law, that is the supplying rate at each level of the supply chain, is presented in the following section.

\subsection{Order rates and control structure}

The order rate $u(t)$ at a given level represents the command of the delayed system given by equations (1). Regarding to the system constraints, and the nature of the system, the control law we propose to apply is a saturated command based on a feedback predictor structure such that

$$
u(t)=\underset{\left[0, U_{\max }\right]}{\operatorname{sat}}\left[K\left(y_{c}-z(t)\right)\right], \text { for } t \geq 0 .
$$

where $y_{c}$ is the reference signal of the system, which corresponds to the reference level for the inventory. $K$ is the controller gain which is used to adjust the order rates placed in each level, and $z(t)$ is the prediction of the future state of the system, that corresponds to the inventory level at $t+\theta$, as it is shown in the sequel.

Saturated commands are commonly used for systems with saturating actuators, and permit to take into account theirs specific limitations. It was shown to be more efficient and realistic than a linear constraint control $[23,9]$. On the other hand, the use of a saturated controller introduces non-linearities in the closed-loop scheme of the system, due to the sat function defined as

$$
\underset{[a, b]}{\operatorname{sat}}[f(t)]= \begin{cases}b & \text { if } f(t)>b, \\ f(t) & \text { if } a \leq f(t) \leq b, \\ a & \text { if } f(t)<a .\end{cases}
$$

For such non-linear systems, stability conditions can be obtained by computing invariant sets in which the system trajectory remains, and in which the saturation constraints are met, as it is shown in the sequel. The feedback predictor part of the command, is used to handle the delays and the stability properties of the infinitedimensional system, by allowing the assignment of the closed-loop system poles, in a finite number of locations in the complex plan $[12,11]$. Also known as model reduction or Artstein reduction [1], the basic idea of state prediction is to compensate the time delay $\theta$ by generating a control law that enables one to directly use the corresponding delay-free system, thanks to the prediction defined by 


$$
z(t)= \begin{cases}y(t)+\int_{t-\theta}^{t} u(\tau) d \tau & \text { for } t \geq \theta, \\ y(t)+\int_{t}^{\theta} \phi(\tau) d \tau+\int_{0}^{t} u(\tau) d \tau & \text { for } t<\theta\end{cases}
$$

which can be rewritten, using expression (1) as

$$
z(t)=y(t+\theta)+\int_{t}^{t+\theta} d(\tau) d \tau, \text { for } t \geq 0
$$

Indeed, by time derivation of this equation (7), one can see that the resulting system

$$
\dot{z}(t)=u(t)-d(t), \text { for } t \geq 0,
$$

is delay-free. The system (8) is the reduced model of the system (1)-(5). Artstein [1] demonstrated that the control low $u(t)$ is admissible for the closed loop system (1)-(5) if and only if it is admissible for the system (8)-(5), and that the two systems have the same dynamics properties. Our approach is then based on the use of the reduced system (8) to design the controller such that the system constraints and requirements (2) and (3) will be fully met, as shown in Section 3.2.

\subsection{The closed-loop system dynamics}

The dynamics of the closed-loop system (8)-(5) is given by the following expression.

$$
\dot{z}(t)=\underset{\left[0, U_{\max }\right]}{\operatorname{sat}}\left[K\left(y_{c}-z(t)\right]-d(t), \text { for } t \geq 0 .\right.
$$

The stability analysis of this system is performed by computing an invariant interval for the trajectories of system (9), in which the system constraints are met, and the BIBO stability property of the system is warranted. In this sake, the system constraints (2) and (3) are reformulated in terms of the new state variable $z(t)$ as follows. Using the expression (7), one can see that

$$
y(t+\theta)=z(t)-\int_{t}^{t+\theta} d(\tau) d \tau, t \geq 0 .
$$

The constraint (2) is verified if both $z(t)$ and the term $\int_{t}^{t+\theta} d(\tau) d \tau$ are bounded, so that $y(t+\theta) \in\left[0, Y_{\max }\right]$. Provided condition (4) is satisfied, it is seen that $\int_{t}^{t+\theta} d(\tau) d \tau \in\left[0, \theta d_{\text {max }}\right], \forall t \geq 0$. Thus, $z(t)$ should be limited by a lower and an upper bound, $z_{\min }$ and $z_{\max }$ respectively, which verifies the relation (10), such that

$$
0 \leq z_{\min }-\theta d_{\max } \text { for } y(t+\theta)=0, \quad \text { and } \quad z_{\max } \leq Y_{\max } \text { for } y(t+\theta)=Y_{\max } .
$$

Then, the original delayed system verifies $y(t) \in\left[0, Y_{\max }\right]$ for all $t \geq 0$, if and only if the condition

$$
z(t) \in\left[\theta d_{\max }, Y_{\max }\right]
$$


with $\theta d_{\max }<Y_{\max }$, is verified for the reduced system. The control problem established in Section 2, is reformulated in terms of founding the controller parameters, which permits to the closed loop system (8)-(7), to verify the constraints (11) under disturbance effects of $d(t)$. The results are given in Section 4.

\subsection{Admissible initial conditions}

Non-zero initial conditions does not affect the control structure and the system constraints. Indeed, as shown by equation (1) for $t \in[0, \theta]$, the inventory level evolution depends on the function $\phi(t)$ and the demand only. Because of the delay, the effects of the command $u(t)$ on the system dynamics are not visible before $t=\theta$. Then, checking whether the system constraints are met or not on the time interval $[0, \theta[$ yields a set of admissible initial conditions, for which the constraint conditions are verified. This set is characterized as follows. Using equation (1), for $0 \leq t<\theta$, the inventory level is given by

$$
y(t)=y_{0}+\int_{0}^{t} \phi(\tau) d \tau-\int_{0}^{t} d(\tau) d \tau,
$$

where $y_{0}$ is the initial inventory level at time $t=0$, and the amount $\int_{0}^{\theta} \phi(\tau) d \tau$ represents the initial WIP in the pipeline that is denoted wip $p_{0}$. It is seen that the term $\int_{0}^{t} d(\tau) d \tau$ belonging to the interval $\left[0, \theta d_{\text {max }}\right], y(t)$ verifies $y(t) \in\left[0, Y_{\text {max }}\right]$ for $t \in[0, \theta[$ if and only if the initial conditions are such that

$$
\theta d_{\max } \leq y_{0}+\text { wip }_{0} \leq Y_{\max }
$$

\section{Controller designing issues}

The controller design consists in determining suitable gain $K$ and inventory reference level $y_{c}$ for each elementary stage of the supply chain, such that the system constraints and specifications are fully met.Two main issues are to be considered. First, for given systems parameters, namely the maximum capacities $U_{\max }$ and $Y_{\max }$, is it possible to find a controller which will fully meet the constraints and the system requirements. Then, if such a controller is indeed feasible, the second issue is about the choice of the command parameters $K$ and $y_{c}$ under the system constraints and specifications. This is the parameterization phase. In this section, both issues are treated through the dynamics properties analysis of the system, such that the exact solution of the equation (9) is not required. Our proposal is to determine some necessary and sufficient conditions on the controller parameters, to impose the invariance property of the interval (11), so that the BIBO stability of the system and the constraints are all satisfied. These conditions are given through the following 
Theorem 1. A corollary is then formulated, which gives further results concerning the closed-loop system dynamics under Theorem 1 assumptions.

Theorem 1. Being given a system of the form (1), there exists a command of the form (5), for which the system is stable and the constraints (2) and (3) are fulfilled, for any $d(t) \in\left[0, d_{\max }\right]$ if and only if the following conditions hold true

$$
\theta d_{\max }<Y_{\max }
$$

and

$$
d_{\max } \leq U_{\max } .
$$

In addition, if the conditions (12) et (13) are met, the constraints (2) and (3) are satisfied under the control law (5) if an only if the controller parameters are such that:

$$
\theta d_{\max }+\frac{d_{\max }}{K} \leq y_{c} \leq Y_{\max }
$$

Proof. As shown in Section 3.2, the controller of the reduced system (9) should be designed such that constraint (11) is fulfilled. The existence of the controller is then linked with the non-empty property of interval $\left[\theta d_{\max }, Y_{\max }\right]$, which is true only when $\theta d_{\max }<Y_{\max }$. This later shows the necessity of condition (12), its sufficiency being obvious.

Conditions (13) and (14) come from the fact that, verifying constraint (11) at any time $t \geq 0$ implies that, the closed interval $\left[z_{\min }, z_{\max }\right]$ is invariant for the system trajectories. Formally, this property is warranted if and only if the following implications are true, for all $t \geq 0$

$$
z(t)=z_{\min } \Rightarrow \dot{z}(t) \geq 0, \quad \text { and } \quad z(t)=z_{\max } \Rightarrow \dot{z}(t) \leq 0 .
$$

Using expression of $\dot{z}(t)$ given by (9), and provided that (4) is true, these inequalities are rewritten respectively

$$
\underset{\left[0, U_{\max }\right]}{\operatorname{sat}}\left[K\left(y_{c}-z_{\min }\right)\right] \geq d_{\max }
$$

and

$$
\underset{\left[0, U_{\max }\right]}{\operatorname{sat}}\left[K\left(y_{c}-z_{\max }\right)\right] \leq 0
$$

Using the sat function definition given in Section 3.1, one can see that the inequality (15) is solvable if and only if $U_{\max } \geq d_{\max }$, that shows the sufficiency and necessity of condition (13), and thus $y_{c}$ is such as $z_{\min }+d_{\max } / K \leq y_{c}$, which, together with condition (12) and the equality $z_{\min }=\theta d_{\max }$, establishes the sufficiency of the left part of the condition (14) of Theorem 1. Its necessity comes form the fact that for $y_{c}<z_{\min }+d_{\max } / K$, inequality (15) has no solution.

The same analysis is applied for inequality (16). This latter is solvable if and only if

$$
y_{c} \leq z_{\max },
$$


which, together with equality $z_{\max }=Y_{\max }$ shows that $y_{c} \leq Y_{\max }$. This establishes the sufficiency and the necessity of the right member of condition (14) of Theorem 1, and completes the proof.

Under the conditions of Theorem 1, the analysis of the closed-loop system dynamics shows that the system constraints and specifications are truly met. Any satisfactory controller actually permits to fill more restrictive constraints on the system variables. We describe these restrictions in the following corollary.

Corollary 1. Being given a system of the form (1), with a control law of the form (5) and suitable initial conditions, such that the conditions (12), (13) and (14) are verified, then the following holds true

$$
y(t) \in\left[y_{c}-\frac{d_{\max }}{K}-\theta d_{\max }, y_{c}\right],
$$

and

$$
u(t) \in\left[0, d_{\max }\right],
$$

for all $t \geq 0$ and $d(t) \in\left[0, d_{\max }\right]$.

Proof. From expression (9), one can observes that, under Theorem 1 assumptions, the following implications are true for all $t \geq 0$,

$$
z(t) \geq y_{c} \Rightarrow \dot{z}(t) \leq 0 \quad \text { and } \quad z(t) \leq y_{c}-\frac{d_{\max }}{K} \Rightarrow \dot{z}(t) \geq 0
$$

These implications show that the effective interval of variation of $z(t)$ is such that

$$
z(t) \in\left[y_{c}-\frac{d_{\max }}{K}, y_{c}\right],
$$

which represents the smallest invariant interval for the system (9). Indeed, under Theorem 1 assumptions, it is seen that the interval given in (20) is included in the interval given by (11). Thus, using expressions (10) and (5), one can compute the effective interval of $y(t)$ and $u(t)$ variations which are given by expressions (18) and (19) respectively.

\section{Generalization for N-stages supply chain}

In this section, we propose a generalization of the results presented above for the multi-stages supply line, composed of $\mathrm{N}$ elementary stages, as presented in Section 2. Each level is now labelled with a subscript $i$, with $i=1, . ., N$. In such serially linked structure, each stage $i$ has one supplier $i-1$, and is supposed to support the incoming demand $d_{i}(t)$ of the following stage $i+1$ such that $d_{i}(t)=u_{i+1}(t)$ for $i=1, . ., N-1$. The last stage $N$ of the supply chain is usually the final retailer 
which faces the end consumer demand denoted $d_{c}(t)$. The inventory dynamics of each stage is given by the following equation.

$$
\dot{y}_{i}(t)= \begin{cases}u_{i}\left(t-\theta_{i}\right)-d_{i}(t) & \text { for } t \geq \theta_{i}, \\ \phi_{i}(t)-d_{i}(t) & \text { for } 0 \leq t<\theta_{i},\end{cases}
$$

where, $y_{i}(t)$ is the inventory, $u_{i}(t)$ is the acquisition rate with delay $\theta_{i}$, and $d_{i}(t)$ the incoming demand, with $i=c$ for the customer demand rates.

The present part aims to define the controller parameters of each stage, such as the end consumer demand $d_{c}(t)$ will be satisfied, and taking into account the local constraints of each single level, as seen in Section 4, and the additional constraints due to the serial structure as well. The constraints (2) and (3) are generalized as follows. For $i=1, . ., N$, the inventory levels are such that

$$
y_{i}(t) \in\left[0, Y_{\text {max }_{i}}\right], \text { for } t \geq 0,
$$

and the acquisition rates verify

$$
u_{i}(t) \in\left[0, U_{\text {max }_{i}}\right], \text { for } t \geq 0 .
$$

The additional constraints arising from the network structure are about the incoming demand of each level, where $d_{i}(t)=u_{i+1}(t)$ for $i=1, . ., N-1$, such that

$$
d_{i}(t) \in\left[0, U_{\max _{i+1}}\right] .
$$

For the retailer stage $i=N$, the incoming demand is the end customer demand $d_{c}(t)$. It verifies the same assumption (4) namely

$$
d_{c}(t) \in\left[0, d_{\max }\right] .
$$

The same control law as the one presented in Section 3.2, is used in each stage. It is of the form

$$
u_{i}(t)=\underset{\left[0, U_{\max _{i}}\right]}{\operatorname{sat}}\left[K_{i}\left(y_{c_{i}}-z_{i}(t)\right)\right] \text { for } t \geq \theta_{i},
$$

with $y_{c_{i}}$ is the reference level for the inventory $y_{i}, K_{i}$ is the controller gain which is used to adjust the order rates placed in level $i$, and $z_{i}(t)$ is the prediction of the future state of the system, defined as follows

$$
z_{i}(t)= \begin{cases}y_{i}(t)+\int_{t-\theta_{i}}^{t} u_{i}(\tau) d \tau & \text { for } t \geq \theta_{i}, \\ y_{i}(t)+\int_{t}^{\theta_{i}} \phi_{i}(\tau) d \tau+\int_{0}^{t} u_{i}(\tau) d \tau & \text { for } t<\theta_{i},\end{cases}
$$

The controller design issue in each stage is addressed as shown in Sections 3 and 4. Using the same arguments basing on the analysis of the reduced model obtained for each stage, Theorems 1 is now extended to the $\mathrm{N}$-stages supply line.

Theorem 2. Being given a supply chain of the form (21), there exists a command of the form (26), for which the system is stable, and fulfilled the constraints (22), (23) 
and (24), for any $d_{c}(t) \in\left[0, d_{\max }\right]$ if and only if the following conditions hold true.

$$
\theta_{i} d_{\max }<Y_{\max _{i}}
$$

and

$$
d_{\max } \leq U_{\text {max }_{i}}
$$

for all $t \geq 0$ and $i=1, . ., N$. In addition, if conditions (28) and (29) are verified, the constraints (23), (24) and (25) are met for any $d_{c}(t) \in\left[0, d_{\max }\right]$, if and only if the controller parameters are such that:

$$
\theta_{i} d_{\max }+\frac{d_{\max }}{K_{i}} \leq y_{c_{i}} \leq Y_{\max }
$$

For all $t \geq 0$ and $i=1, . ., N$.

Proof. For the N-stages supply line serially linked, the whole supply line dynamics is driven by the end-costumer demand $d_{c}(t)$. Applying Theorem 1 for the last stage $N$ of the line, Theorem 2 shows that the orders $u_{N}(t)$ vary in the interval $\left[0, d_{\max }\right]$. Then the linking relation between the supply line stages, where $d_{N-1}(t)=u_{N}(t)$ shows that actually $d_{N-1}(t) \in\left[0, d_{\max }\right]$. Thus, by recursion, it is seen that $d_{i}(t) \in$ $\left[0, d_{\max }\right]$ for all $i=1, \ldots, N$. Using this result, the demonstration of Theorem 2 is directly derived from the proof of Theorem 1 .

\section{Simulation example and discussions}

The application example presented in this section, aims at highlighting the efficiency of the distributed control scheme proposed to eliminate the bullwhip effect in a supply chain, and to illustrate the importance of taking into account the positivity and capacity constraints. For this sake, we consider a three-stages supply chain, commonly used in the literature [3,8], consisting of a production plant, a wholesale stage, and a distribution centre. Unlike the aforementioned works, where capacity constraints are assumed for the order rates only, we consider both inventories limitations, and positivity constraints. The subscripts $p, w$, and $r$ are used to label the production, the warehouse and the retailer stages respectively. The maximum capacities $U_{\max _{i}}$ and $Y_{\text {max }_{i}}$ and some admissible initial conditions are given in Table 1.

Table 1 Simulation parameters for the constrained three-stages supply chain.

\begin{tabular}{lllllll}
\hline Stage & $K_{i}$ & $\theta_{i}$ & $U_{\max _{i}}$ & $Y_{\max _{i}}$ & $y_{c i}$ & $y_{0 i}+w_{i p_{0 i}}$ \\
\hline$i=p$ & 0.6 & 6 & 350 & 1900 & 1900 & 1900 \\
$i=w$ & 0.6 & 3 & 300 & 1200 & 1200 & 1200 \\
$i=r$ & 0.6 & 2 & 250 & 900 & 900 & 900 \\
\hline
\end{tabular}


The initial conditions are chosen such that all transitory dynamics are avoided, according to the rule defined in Section 3.3. Controller parameters $K_{i}$ and $y_{c i}$ are calculated according to Theorem 2, and are also sorted in Table 1. In order to illustrate the inventory dynamics, the customer demand used for this simulation is a square function starting at $t=15$ weeks and ending at $t=45$ weeks, with an amplitude $d_{\max }=240$, unlike the aforementioned works where only step function demands where considered. The results of the simulation are depicted on Figure 2, where the order rates of each stage and the inventory levels are represented.

Figure 2(a) shows that the order rates placed in each of the three stages, follows closely the demand, causing no amplification through the upstream levels as it is expected by the relation (24). Then, Figure 2(b) shows that the inventory levels remain non-negative, and are re-completed to their reference levels when the demand is null, as it was specified by the ordering policy presented in Section 1. The outcomes of this simulation study, join the former authors conclusions [8, 29, 2, 3] which stand that capacity constraint of the ordering rates, does not necessarily impact the customer service level, which corresponds to the demand satisfaction. The saturating constraint impacts the dynamics too, such that the order rates being limited, the supplying process completion takes more time, but since the condition (29) is verified, the demand is always fulfilled. It is also recognized that such constraints provide an effective improvement in reducing the demand amplification, within the multi-echelon system. Indeed, we showed that a good handling of the delays, via

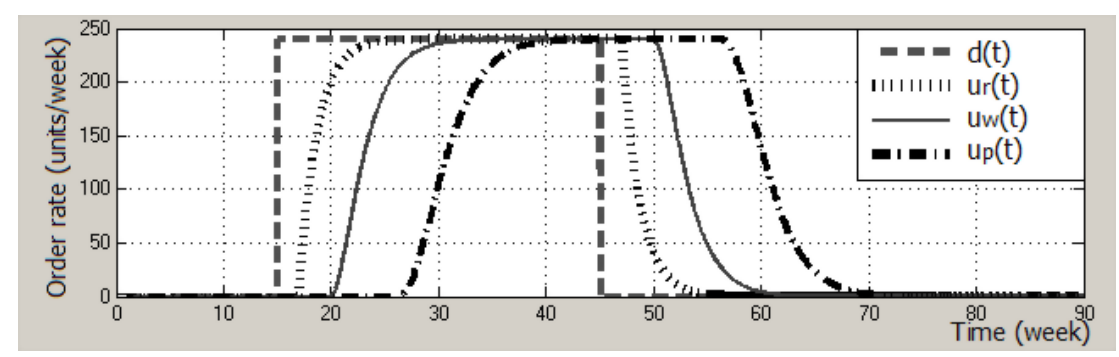

(a) Dynamics of the order rates

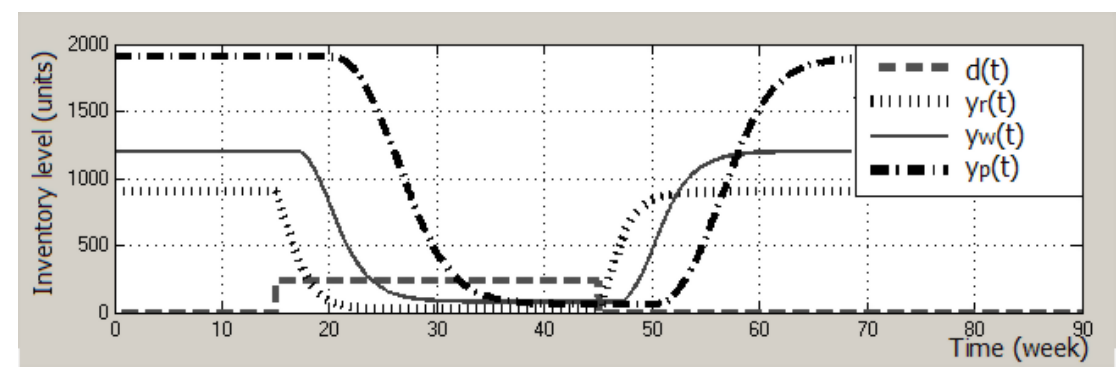

(b) Dynamics of the inventory levels

Fig. 2 Simulation results for the constrained three-stages supply line. 
an appropriate control law, permits to definitely overcome the Forrester effect. This result is also pointed out by the former work of [18] and [16], where it is established that the smoothest system responses are obtained when the same care is given to the inventory discrepancy and the WIP. The formal explanation of this empiric result comes from the input time delay system control, as seen in Section 3, where the efficient delay compensation via the predictor feedback imposes the same coefficient $K$ for both the inventory discrepancy and the distributed delay of the predictor which is the WIP term. Assuming an unknown bounded demand as a working assumption, allows us to maintain this results for every bounded demand signal, no mater if it is a step function shaped or not.

\section{Conclusion and perspectives}

In this chapter, the controller design problem for serially-linked supply chains, with constrained orders and inventories, and unknown customer demands variations, has been investigated. The problem is stated in terms of controlled input time delay system, with positivity and saturations constraints, subject to bounded disturbances. A saturated feedback predictor controller was introduced to handle both the delayed dynamics and the constraints, where the controller encompasses a distributed delay expressed by the integral term in the prediction. This distributed term corresponds to the WIP amount which the use in inventory regulation is quite classical for damping the bullwhip effect $[10,16,18]$. It is important to notice that the WIP is actually measurable. Thus, the controller proposed in this work is of low complexity, since it corresponds to a static feedback on measurable variables. The main advantage of this work is that practical constraints of positivity and capacity of both orders and inventories are taken into account, that enhanced the accuracy of the results. In addition, the controller proposed eliminates totally the Forrester effect, in case where the delays are properly known. Robustness analysis of the results in case of delay misestimations, and the consideration of variable delays are advised of forthcoming works.

\section{References}

1. Artstein, Z.: Linear systems with delayed controls: A reduction. IEEE Transactions on Automatic Control. 27 (4): 869-879 (1982)

2. Cannella, S., Ciancimino, E. and Mrquez, A. C.: Capacity constrained supply chains: a simulation study. International Journal of Simulation and Process Modelling. 4 (2): 139-147 (2008)

3. Evans, G.N. and Naim, M.M.: The dynamics of capacity constrained supply chains. Proceedings of International System Dynamics Conference, Stirling, Scotland, 28-35 (1994)

4. Delice, I. I. and Sipahi, R.: Inventory dynamics models of supply chains with delays ; systemlevel connection \& stability. In: Loiseau, J. J., Michiels, W., Niculescu, S., and Sipahi, R., 
(eds.) Topics in Time Delay Systems, of Lecture Notes in Control and Information Sciences. Springer, Berlin Heidelberg, 38: 8349-358 (2009)

5. Edghill, J.S., Towill, D.R.: The use of systems dynamics in manufacturing systems. Transaction of the Institute of Measurement and Control. (1989) doi: 10.1177/014233128901100406

6. Forrester, J. W.: Industrial Dynamics. Cambridge MA: MIT press, (1961)

7. Gavirneni, S., Kapucinski, R. and Tayur, S.: Value of information in capacitated supply chains, Management Science. 45 (1): 16-24 (1999)

8. Helo, P.T.: Dynamic modelling of surge effect and capacity limitation in supply chains. International Journal of Production Research. 38 (17): 4521-4533 (2000)

9. Hu,T. L. Z.: Control Systems with Actuator Saturation : Analysis and Design. Birkhäuser, Boston (2001)

10. John, S., Naim, M.M., Towill, D.R.: Dynamic analysis of a WIP compensated decision support system. International Journal of Management Systems and Design. 1 (4): 283-297 (1994)

11. Kwon, W. and Pearson, A.: Feedback stabilization of linear systems with delayed control. IEEE Transactions on Automatic Control, 25 (2): 266-269 (1980)

12. Manitius, A. and Olbrot, A.: Finite spectrum assignment problems for systems with delays. IEEE Trans. Automatic Control. 24: 541-553 (1979)

13. Mirkin, L. and Raskin, N.: Every stabilizing dead-time controller has an observer-predictorbased structure. Automatica. 39 (10): 1747-1754 (2003)

14. J. P. Richard, Time-delay systems : an overview of some recent advances and open problems. Automatica. 39 (10): 1667-1694 (2003).

15. Riddalls, C.E., Bennett, S. and Tipi, N.S.: Modeling the dynamics of supply chains, International Journal of System Science. 31: 969-976 (2000)

16. Riddalls, C. E., et Bennett, S.: The stability of supply chains. International Journal of Production Research. 40 (2): 459-475 (2002)

17. Shukla, V., Naim, M.M.: The impact of capacity constraints on supply chain dynamics. International Conference on Computers \& Industrial Engineering. CIE 2009, 925-930 (2009)

18. Simon, H. A.: On the application of servomechanism theory in the study of production control. Econometrica. 20: 247-268 (1952)

19. Sipahi, R., Delice, I.I.: Supply network dynamics and delays; performance, synchronization, stability. Mechanical and Industrial Engineering Faculty Publications (2008) Available at http://works.bepress.com/rsipahi/13

20. Sipahi, R., Delice, I.I.: Stability of Inventory Dynamics in Supply Chains with Three Delays. International Journal of Production Economics. 123: 107-117 (2010)

21. Sipahi, R., Niculescu, S.-I., Abdallah, C. T., Michiels, W. and Gu, K.: Stability and Stabilization of Systems with Time Delay, Limitations and opportunities. IEEE Control Systems Magazine. 31 (1): 38-65 (2011)

22. Sterman, J. D.: Modelling managerial behaviour misinterpretations of feedback in a dynamic decision-making experiment. Management Science. 35 (3): 321-339 (1989)

23. Tarbouriech, S., Garcia, G., Da Silva, J., and Queinnec, I.: Stability and Stabilization of Linear Systems with Saturating Actuators. Springer (2011)

24. Towill, D.R.: Dynamic analysis of an inventory and order based production control system. International Journal of Production Research. 20 (6): 671-687 (1982)

25. Warburton, R. D. H.: An exact analytical solution to the production inventory control problem. International Journal of Production Economics. 92: 81-96 (2004)

26. Warburton, R. D. H. , Disney, S. M., Towill, D. R. and Hodgson, J. P. E.: Further insights into 'the stability of supply chains'. International Journal of Production Research. 42 (3): 639-648 (2004)

27. Wang, X., Disney, S.M. and Wang, J.: Exploring the oscillatory dynamics of a forbidden returns inventory system. International Journal of Production Economics (2012). ISSN 09255273, http://dx.doi.org/10.1016/j.ijpe.2012.08.013.

28. Wang, X., Disney, S.M. and Wang, J.: Stability analysis of constrained inventory systems with transportation delay. European Journal of Operational Research. 223 (1): 86-95 (2012)

29. Wikner, J., Naim, M.M. and Rudberg, M.: Exploiting the order book for mass customized manufacturing control systems with capacity limitation. IEEE Transactions on Engineering Management. 54 (1): 145-155 (2007) 